\title{
015 NEUROLOGISTS ADHERENCE TO GUIDELINES WHEN PRESCRIBING PLEX
}

Thomas Peukert, ${ }^{1}$ Stephen Hunt, ${ }^{1}$ Gary Benson, ${ }^{2}$ Heather Knipe ${ }^{2} .{ }^{1}$ Royal Victoria Hospital, Belfast; ${ }^{2}$ Belfast City Hospital

\subsection{6/jnnp-2014-309236.15}

Background The number of patients receiving plasma exchange (PLEX) for neurological symptoms in Northern Ireland has increased dramatically. Between January 2009 and September 2013, a total of 59 patients received plasma exchange.

Objectives To determine if Northern Ireland neurologists are compliant with the American Society for Apheresis ( 2013) and the American Academy of Neurology (2011) guidelines, for prescribing PLEX. To identify which diseases responded positively to PLEX treatment.

Methods Patient notes were reviewed $(n=59)$ to determine if PLEX was prescribed in diseases for which there is evidence according to the AAN and ASFA guidelines. Data was extracted to determine which groups of patients improved with treatment, which developed side effects and how this compared to the recommendations in each guideline.

Results Compliance with guidelines-ASFA 84.7\%, AAN $66.1 \%$. Improvements were noted in $71.2 \%$ of patients receiving PLEX, 28.8\% showed no improvement or deteriorated. Conditions that showed good improvement included GBS, CIDP, Myasthenia gravis and NMO. Patients with peripheral neuropathies did not improve after PLEX. Side effects included electrolyte imbalances, PE and DVT (5 patients).

Conclusion The overall compliance rate with either AAN or ASFA guidelines was $93.2 \%$ and the results of PLEX treatment appear consistent with current recommendations. 